\title{
An Efficient Modification of Grey Wolf Optimization Using Cuckoo Search, Levy Fly and Mantegna Algorithm for Real-Time Image Processing Applications
}

\author{
Sayantan Dutta, Ayan Banerjee \\ Faculty of Electronics \& Telecommunication Engineering, IIEST, Shibpur, Howrah-711103
}

ABSTRACT - Optimization methods, frequently used in several image and video processing algorithms for the attainment of optimal solutions, pose severe hurdle in case of real-time processing. For catering to the needs of real-time operations in a cost-effective way, dedicated hardware is inevitable. The huge computational load of any optimization method strikes down its feasibility of being realized in terms of dedicated hardware. The computational complexities of meta-heuristic optimization methods are even more than any other conventional optimization methods. So, in spite of having the capability of providing global solution by dodging local optima, meta-heuristic optimizations are avoided in real-time systems. To overcome the bottleneck, in this article, a modified GWOA (modified grey wolf optimization algorithm) is formulated by blending the advantages of CS (cuckoo search), Levy fly (LV), and MA (Mantegna algorithm). This modified GWOA is articulated to be computationally efficient and precise, so that, it can easily be realized in terms of dedicated VLSI architecture while maintaining the accuracy at a high level. The proposed method helps to diminish the cost and power requirement of high end and costly real-time imaging/ video processing systems while upholding its precision. The proposed method is tested by using MATLAB R2018b. The high-level synthesis (HLS) tool of Xilinx Vivado 18.2 software is used to synthesize this MGWOA, thus establishing the viability of the proposed algorithm to be implemented on FPGA/ ASIC.

\author{
ARTICLE HISTORY \\ Revised: 15 July 2020 \\ Accepted: 19 Feb 2021
}

\section{KEYWORDS}

GWO (grey wolf optimization), $\quad \mathrm{HO}$ (Heuristic optimization), CSA (cuckoo search algorithm), LSA (levy search algorithm), MO (metaheuristic optimization), MA (Mantegna algorithm), prey, Nature-inspired algorithm.

\section{INTRODUCTION}

In the first world countries, there is extensive use of image processing in the major sector, like SI (satellite imaging) and MI (medical imaging). IP (image processing) is used in a different field, and some extensive usages are in image fusion, image imprinting, image registration, image scaling, and many more. Though IP has plenty of applications, its implementation is limited because there is no such dedicated hardware to implement in RTIP (Realtime image processing). For implementing it on dedicated hardware, there is a deficiency of optimum algorithm. Clinical diagnosis plays a big role in medical imaging. In this section, IF (image fusion) and scaling based technique is widely used, but the produced imaged quality is no maintained. By failing, the quality results lead to inappropriate diagnosis. An inappropriate diagnosis in the medical field may cause the risk of a patient's life.

In the field of medical imaging, nature-inspired metaheuristic algorithms are the trending method in recent days. This is used in finding optimum search results. There is a list of algorithms in MHA (meta-heuristic algorithm), like GWO, GSA-PSO, PSO, GSA, and many more. In recent days, among those algorithms, GWO gets huge attention from others. Like other animal wolfs tries to maintain a hierarchical structure where at the top place is dedicated to the leader. The leader assists them in the hunting mechanism. Exploration and exploitation are the two parts of the hunting mechanism of grey wolfs. Exploration is divided into two subparts. One is searching, and another is encircling. In 2014, the GWO algorithm was proposed by Mirjalili et. al [1]. The three major steps of these algorithms are searching, encircling, and attacking. After the GWO algorithm, there are several algorithms that have been proposed, but the popularity of the GWO (grey wolf algorithm) remains the very same. The GWO algorithm can be modified in many ways. In the year 2014, Mirjalili et. al proposed GWO, inspiring from three major steps (searching, encircling, and attacking) [1]. After that, there are many nature-inspired algorithms that have been proposed, but the popularity of the GWO (grey wolf algorithm) remains the same. The performance of the GWO algorithm in terms of computational speed and reliability can be improved. The first way to improve performance is to introduce new operators. So, the results will be more precise and reliable. The second way is to add a different parameter. In that way, the distance of exploration can be modified and control more reliably. In the third way, we can add a different parameter. As a result, hybrid GWO can handle more difficult problems. The position updating equation plays an important role in GWO, which helps GWO to converge more easily, and that can be used as a fourth way for modifying the GWO algorithm. The result of position updating gives better convergence speed. The pictorial view of those four different ways to modify the GWO algorithm is depicted below. 


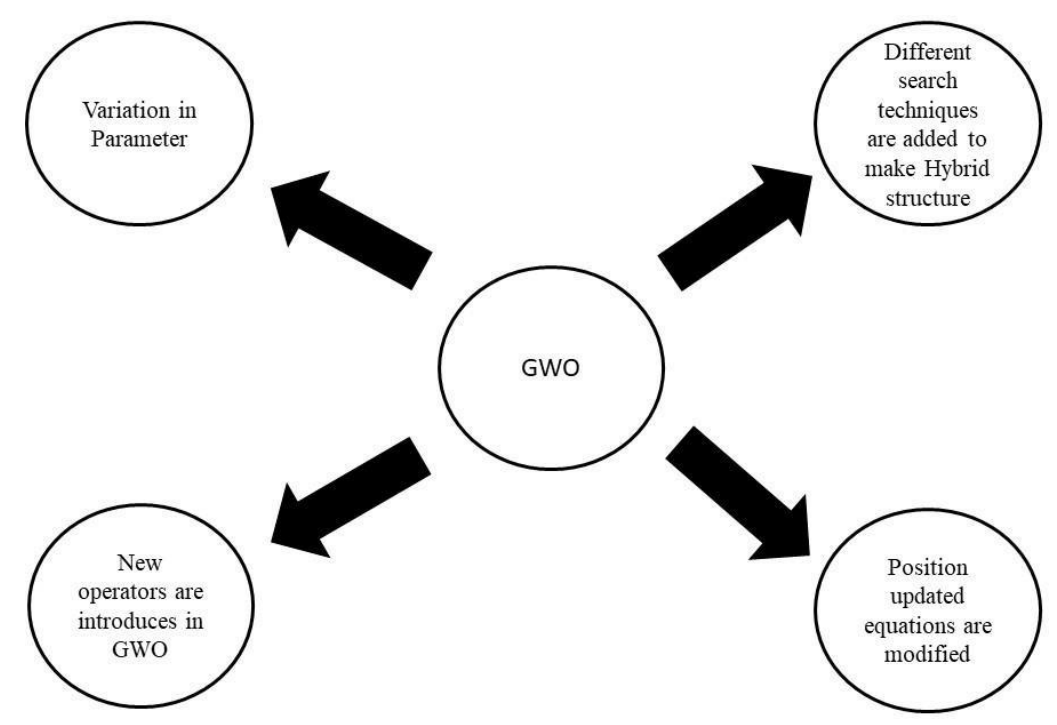

Figure 1. Different ways of modification of GWO in recent days.

Processed images require an in-depth analysis of clinical diagnosis in medical imaging. Erroneous results or approximate algorithms can throw the patient's lives in danger. So, the detection of suspicious objects in the patient's body should be accurate. In a normal scenario, micro cameras are used to capture images inside the patient body. Those micro cameras are mounted onto the probe. Those taken pictures are sent to the doctor for identifying the disease. Depending on those images, doctors try to understand how deadly is the disease is and accordingly prescribed the necessary steps. In this paper, a new kind of algorithm has been proposed. For this reason, we have used CSA (cuckoo search algorithm) because it is an efficient algorithm for finding unknown or known objects. Here fitness function gives us support to detect the most badly affected cell in the human body. LF (Levy Fly) algorithm has been used to guide the camera probe to restrict the random movement. One of the reasons for falling the GWO (grey wolf optimization) is the random movement, which is very critical to handle. From past experience, it is noticed that badly affected cells are situated in the dense region. So, the improvement of camera movement has been made by optimizing the Step size, and in this case, MA (Mantegna algorithm) is used. Our main objective is to find an optimal result. For this reason, we have proposed MGWOA (modified grey wolf optimization algorithm) in this article. In the coming section, the steps of this algorithm to optimize the result has been discussed.

This article is arranged in some sections and further divided into some subsections. First, the introduction part is described in Section I, and the existing work has been described in Section II. The grey wolf optimization is described in Section III. In Section IV cuckoo search algorithm has been mentioned, and in Section V, the levy search algorithm has been described. The detailed discussion of our proposed modified GWO (grey wolf optimization) algorithm is depicted in Section VI. The result of the GWO algorithm and comparison with other algorithms is mentioned in Section VII. In the last section, the GWO algorithm has been concluded (Section VIII).

\section{RELATED WORK}

Last few decades, researchers have tried hard and sold to modify the optimization process. One of the significant improvements they have made is in the grey wolf optimization algorithm. The most critical phase of the metaheuristic algorithm is the searching process. In resect days, researchers have blends the heuristic algorithm with the metaheuristic algorithm to detect objects easily by improving the searching process. Some notable improvement in the CSA algorithm, GWO algorithm, and image fusion methods in recent days is described in the section.

Using of Image fusion in the field of medical imaging is growing up day by day. If we specifically say, then it is mainly used in clinical diagnosis. In the case of MIF (multimodal image fusion), scaling based technique is hugely used, but in normal scaling, the quality of image fusion is degraded due to the selection of static scaling value. In this context, Daniel et. al [2] have used the Grey Wolf Optimization algorithm (GWO) in optimum spectral mask fusion (OSMF) in their first paper. This OSMF is used in medical image fusion. Before that, in multimodal image fusion, the single scaling-based technique is used. The main drawback of this generalized scaling-based technique is that it reduces the quality of fusion. So, for correcting image and edge quality, the spectral mask technique gives a better result than regular special domain transformation. Thus, they have achieved more information in multimodal image fusion [2].

IN MMMIF (multimodal medical image fusion) MSMT (multi-scale masking technique) is a widely used technique. In medical imaging for image fusion, our main concern is the quality of components information. DWT (discrete wavelet 
transform) is a well-known process in image fusion for MMMI (multimodal imaging), but the drawback is that the approximation percentage is greater. As a result, less edge feature is available. Contradictory with the DWT Laplacian filter is better because it provides more edge features. In that regard, the second paper of Daniel et. al [3] have done a Laplacian wavelet mask in medical imaging. This is achieved by using a hybrid cuckoo search, which is incorporated with the grey wolf algorithm. In medical imaging toughest task is to retain the important information of the component. Previously they have achieved by using multimodal image fusion technique, but it has two drawbacks. First, it approximates greater than is required and secondly a lesser quantity of edge feature available. In their second paper, they have achieved better edge features by using the Laplacian filter-based Technique. In their paper, they have found the control parameter by using the cuckoo search algorithm [3].

In medical imaging, the main concern is to reduce the cost of sophisticated equipment. In that scenario, image fusion is a great choice. It provides adequate information by which clinical diagnosis can be made easily. In this regard, Daniel has done Wavelet-based homomorphic image fusion in his third paper [4]. He has achieved this by using the grey wolf optimization algorithm (GWO). In a typical homomorphic filter for image input, Fourier domain feature is used, but in a multimodal medical image, compare to Fourier spectrum discrete wavelet transform based technique gives better result in terms of greater features. In this paper, he has done OHWF (optimum harmonic wavelet fusion) by using HG-GWO (hybrid genetic - grey wolf optimization). In his previous article for image input, he has used the wavelet-based homomorphic fusion, but in this paper, for image input, he has used logarithmic as well as wavelet domain information [4].

Cuckoo is one of the most used nature-inspired algorithms in recent days. CS falls under swarm intelligence, which is the MHOA (metaheuristic optimization algorithm). This type of optimization technique has a broad class of applications because it gives an excellent result. If the fitness function is large, then CS requires a large number of computations. As a result, efficiency and speed are reduced. To overcome this problem, Alfailakawi et. al have implemented cuckoo search optimization in FPGA. Inspiring from meta-heuristic optimization and their broad application of the problem-solving approach, they have implemented CSO in FPGA. In this paper, they have tried to give a general approach to solve computationally intensive work in less time using by parallel pipelined based field programmable based gate array approach, and their outcome is $75 \mathrm{x}$ time faster than software-based implementation [5].

In the case of function optimization CS (cuckoo search) has great efficiency. Except that it is used in FFNN (feedforward neural network). Having some advantages like fewer parameters and simple calculation Rui-min JIA et. al have used local search with the complex-valued CSA. In the case of function optimization, CSF (cuckoo search function) have many defects in terms of low accuracy, slow speed, and trapping of local minima. By facing such a problem in CSO, they proposed CVCS (complex-valued cuckoo search) with the help of local search, and this approach is well suited for large dimensional optimization problems, and it will increase the capacity of information. In this article, by using a complexvalued function, population diversity is increased. They have used local search at the end of CS. So, that speed will increase significantly [6].

The objective of the multi-focus system is to focus all the focus point in the image. This can be done by combining multiple focused frames of the same image. So, Fu et. al proposed a multi-focus image fusion method that is based on the DCS (distributed compressed sensing). First, the source image is compared in terms of high and low-frequency images. Then depending on that, a dictionary of high and low frequency is created. Then they have used distributed compressed sensing in the JSM-1 (joint sparsity model-1). So, high-frequency images can be reconstructed. In the third stage, they have obtained a decision map from the high-frequency images. This can be further improved by morphological processing. At the end, using the source image, a focused image is taken based on the decision map [7].

In a visual system like the military, tracking and surveillance, infrared image fusion, and visual image fusion are often used. In this article, Zhang et. al have tried to build a new type of infrared image fusion and visual image fusion technique. To do that, they have used NSCT (nonsubsampled contourlet transform) and fuzzy sets. By using NSTC, pre-registered imaged are transformed into high and low-frequency sub-bands. In this regard, they have introduced a new rule using intuitionistic fuzzy that is dedicated to the high-frequency sub-band. On the other hand, an energy-based fusion rule has been used in the case of low-frequency sub band. Inverse NSCT has been used to find the fused image [8].

Outdoor image visible visual quality is hugely affected by the haze. In real-life, the haziness of the captured image can be removed by using the image dehazing technique. Global image saturation and contrast can be enhanced by a normal dehazing algorithm that is present in the market, but in that case, local enhancement has been ignored. So, the produced image cannot offer adequate visual performance in terms of local details. Zheng et. al have proposed an image dehazing solution using PADMEF (position integrated multi exposed image fusion) technique. First, from the SMI (single blued image) underexposed image sequence has been extracted. On the extracted image, gamma correction has been performed, followed by SLA (spatial linear adjustment). Then the previously obtained images are fused to get a haze-free image by using the MEF (multi-exposer image fusion) technique. This technique can be used to remove the degradation made by haze. In this technique, we do not require to use the depth mapping technique [9].

The most popular image enhancement technique is gamma correction. The problem is that in the high illuminated region, there is low contrast. The technique to improve the dark visible region as well as to improve the high contrasted brighter area in the low light images has been discussed in this paper. So, Li et. al proposed a new method named PCGF (pair of complementary gamma functions) technique by using image fusion. PCGF has the potential to provide 
outstanding low light image enhancement. To compare the effectiveness and performance, they have designed a low light image enhancement method. This has been done by a redesigned fusion mechanism. By using an enhanced image, two images are derived for fusion. This has been done by the shipping method and the PCGF method [10].

\section{GREY WOLF OPTIMIZATION:}

- One of the most popular nature-inspired algorithms is GWO (grey wolf optimization). GWO follows the hierarchical structure, and it is inspired by the wolf's social behavior. In this section, we have discussed the behavior of wolves and how it impacts the optimization process.

- Inspiration: In the food chain, a grey wolf is positioned at the top of the pyramid because they are carnivorous. Generally, 5 to 13 wolves move together as a group. This group of wolves together performs the hunting. SO, it is called group hunting behavior. In the group of a grey wolf, a different number of search agent can be present. Depending on the number, strength is defined, and according to the strength, hierarchically, they are divided into subgroups. In general, depending on the fitness value, wolves are divided into four subgroups.

- $\alpha$ (Alpha)

- $\beta$ (Beta)

- $\delta$ (Delta)

- $\omega($ Omega)

The social domain hierarchy has been shown in the figure below.

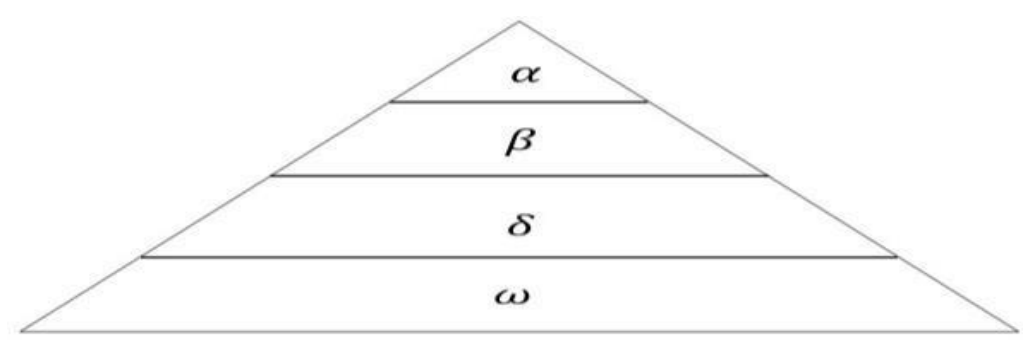

Figure 2. Social domain hierarchy of GW (Dominance decreases from top to bottom).

Searching behavior: Wolf hunting behavior is known as group hunting, and GWO (grey wolf optimization) follows the same mechanism for searching part. This is fast suggested by Mirjalili \& Lewis in 2014 [1]. Exploration and exploitation are two phases of GWO (grey wolf optimization). This can be further subdivided into three subphases. Those are searching, encircling, and attacking.

- Searching: Searching is the subphase of one of the main phase, that is exploration. In this subphase, the prey is searched by wolves.

- Encircling: In this phase, wolves surround the prey after hunting. This is also the subphase of the main phase exploration.

- Attacking: This is the subphase of main phase exploitation. Here $\alpha$ wolves guide $\beta$ wolves, and $\beta$ wolves guide $\delta$ wolves.

In the first step of iteration best three solutions $\left(\alpha_{1}, \beta_{1}\right.$, and $\left.\delta_{1}\right)$ are recorded. This helps to update the position if subordinate wolves.

\section{Exploration}

I.a. Searching: The position of $\alpha, \beta$, and $\delta$ wolf decide the searching operation. Their first operation is diversification from each other at maximum distance. This step is necessary to search and select the prey. After finding the prey, they converge, so that region can be concentrated to attract the prey. GWO's searching method is modeled using random value. The value of ' $A$ ' is used to control the distance. Depending on that value, the wolves either diverge or converges. The wolves will diverge if the A's value is greater than 1. Then they will find suitably positioned prey to attack [11].

I.b. Encircling prey: Suitable prey is encircled at the time of hunting behavior. In GWO, this HV (hunting behavior) is mathematically recorded using equations (1) and (2).

$$
\vec{D}=\left|C \cdot \stackrel{M}{X}_{p}(t)-X(t)\right|
$$




$$
X(t+1)=\overleftrightarrow{X}_{p}(t)-. \vec{D}
$$

$\mathrm{CI}$ (current iteration) is denoted by ' $\mathrm{t}$ ', where $A$ and $C$ defines coefficient vectors. At the position where the prey is located, that position is listed by $\overleftrightarrow{X}$. The grey wolf position is recorded. In the equation (3) and (4), $A$ and $C$ 's definition is declared [12].

$$
\begin{aligned}
& =2 \cdot a \cdot \vec{r}_{1}-a \\
& =2 \cdot \vec{r}_{2}
\end{aligned}
$$

$a$ 's value is declared in the range from 0 to 2 over the course of an iteration. $\vec{r}_{1}$ and $\vec{r}_{2}$ are random variables. Their range varies between 0 and 1 .

From equations (1) and (2), it is clear they affect neighbors as well as two-dimensional position vectors. Initially, preys are situated at the location $\left(X^{*}, Y^{*}\right)$. GW (grey wolves) are located at $(X, Y)$, and their position is dependent on the position of prey [13]. In a pack of a grey wolf, the best-positioned wolf decides the next position of other wolves. By updating the value of $A$ and $C$ to a new value will mathematically update the current position, and the position $\left(X^{*}-X, Y^{*}\right)$ can only be reached by putting the value $A=(1,0)$ and $C=(1,1)$ in the equations.

$\vec{r}_{1}$ and $\vec{r}_{2}$ are the random variables, and by changing those values, wolves can move any suitable position to attack the prey. If a circle is considered as movement space, then GW (grey wolf) can easily locate and relocate their position by encircling the prey, and this can be done by equations (1) and (2).

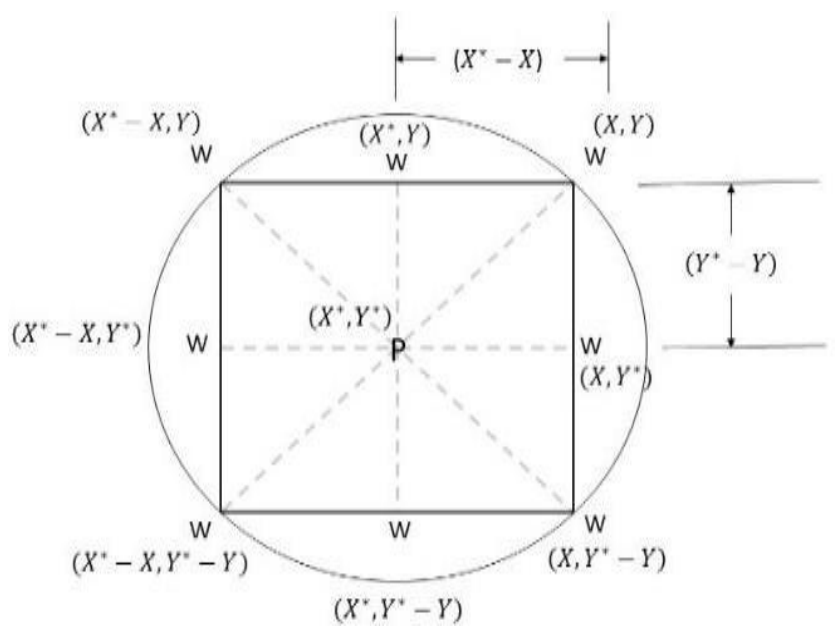

Figure 3. Updated position of grey wolves in 2-D space [1]

\section{Exploitation}

II. a. Attacking: The hunting behavior is started by a superior wolf, that is the $\alpha$ wolves and the subordinate wolves $\beta$, and $\delta$, blindly follows the order of $\alpha$ wolves. It often happens that subordinate wolves participate in the hunting process. In a predefined SP (search space), prey can be located at any position. SO, the wolf has no idea about the position of the prey. Depending on natural tendency like $\alpha$ has better-recognizing capability and $\beta$, and $\delta$ follow the command, and the GWO algorithm is structured mathematically. So, it can produce an as closest result as GW (grey wolf). First, the position of all the wolves is calculated mathematically. From that mathematical solution, the best three solutions are picked up. The other wolf's position is updated, depending on the best solution. Equation (5) to (11) describe the way to find the best solution.

$$
\begin{gathered}
\overrightarrow{D_{\alpha}}=\left|\overrightarrow{C_{1}} \cdot \overrightarrow{X_{\alpha}}-\vec{X}\right| \\
\overrightarrow{D_{\beta}}=\left|\overrightarrow{C_{2}} \cdot \overrightarrow{X_{\beta}}-\vec{X}\right| \\
\overrightarrow{D_{\delta}}=\left|\overrightarrow{C_{1}} \cdot \overrightarrow{X_{\delta}}-\vec{X}\right| \\
\overrightarrow{X_{1}}=\overrightarrow{X_{\alpha}}-\overrightarrow{A_{1}} \cdot\left(\overrightarrow{D_{\alpha}}\right) \\
\overrightarrow{X_{2}}=\overrightarrow{X_{\beta}}-\overrightarrow{A_{2}} \cdot\left(\overrightarrow{D_{\beta}}\right) \\
\overrightarrow{X_{3}}=\overrightarrow{X_{\delta}}-\overrightarrow{A_{3}} \cdot\left(\overrightarrow{D_{\delta}}\right) \\
\vec{X}(t+1)=\frac{\overrightarrow{X_{1}}+\overrightarrow{X_{2}}+\overrightarrow{X_{3}}}{3}
\end{gathered}
$$




\section{CUCKOO SEARCH ALGORITHM}

CSA: In 2009 CSA algorithm has been proposed by Yang \& Deb [14]. Depending on the natural behavior of cuckoo, this algorithm has been built. CSA is a metaheuristic-based optimization technique. Cuckoos generally lay their eggs in other host bird's nests. If the host birds are unable to detect the cuckoo eggs, then the host bird hatches the cuckoo eggs, and the cuckoo chicks come out from the host bird's nests. This natural tendency of cuckoo is mimicked to optimize the search algorithm [14].

We know that cuckoo birds lay their eggs in the host bird nests. Cuckoo birds first select the host bird nests. Then the main job of female cuckoo birds is to go to every selected host bird nest and mimic the color, pattern, and size. This is an important step; in this step, the chance of rejection of cuckoo egg in the other bird nest is decreased. So, productivity is increased. By chance, host birds identify that some eggs differ from their own eggs, then there are two ways to choose decision. The first way is that they can throw the cuckoo egg from their own nest. The second way is that they can move from their own nest and build their nest in a new place [15]. Cuckoo birds select the host bird nests in a way that they recently laid their eggs in their nest. Cuckoo bird eggs are hatched by the host birds slightly earlier than host bird eggs. When the cuckoo bird babies come out from their eggs, their first job is to throw the host birds eggs. They do this action because it increases the probability of getting food from the host birds. It is found from a study that the cuckoo chicks imitate the host birds call that increases the probability of getting food from host birds. Every egg defines a solution, and the cuckoo egg gives a new solution. The main motive of cuckoo birds is to change their own eggs with the host bird egg. The strategy to replace the host bird egg is that the cuckoo bird egg should be superior to the host bird egg. For simplicity of the problem, we first assumed that in every nest, there is one egg. This can be extended further where we can take more number of eggs in every nest. At that time, we will find a set of solutions [16-19].

CSA is a metaheuristic optimization process, and it has three main rules. First, the cuckoo birds choose the host bird nest randomly and lay their eggs. The second rule is that the fittest egg will go to the next generation. The third rule is that the host bird nest is constant. Host birds may or may not detect the cuckoo bird eggs, and the probability is $P_{a} \in$ $[0,1]$. If the host bird identifies the cuckoo bird egg, then there are two possibilities [20]. That is depicted in the picture below. Here compare with the host bird nest, first $\mathrm{n}$ general initial population is generated. Then cuckoo birds choose $\mathrm{k}$ nest randomly. Host birds nest contain their own eggs. Cuckoo birds observe those nests and try to find the nearest similarity with their own eggs to the host bird nests. This comparison of choosing the appropriate nest for laying their eggs depends on the color and fitness of cuckoo's eggs with the fitness of host birds eggs. After completion of this process, host birds come and notice that their own eggs are healthy or not. If they find any dissimilarity of their own eggs in terms of fitness value, then they make a tough decision. If the eggs' fitness value is less than their own laid eggs, then they throw those eggs, and only higher valued eggs can sustain. In the other scenario, if they see the fitness value of most of the eggs are less, then they leave that nest and built a new nest.

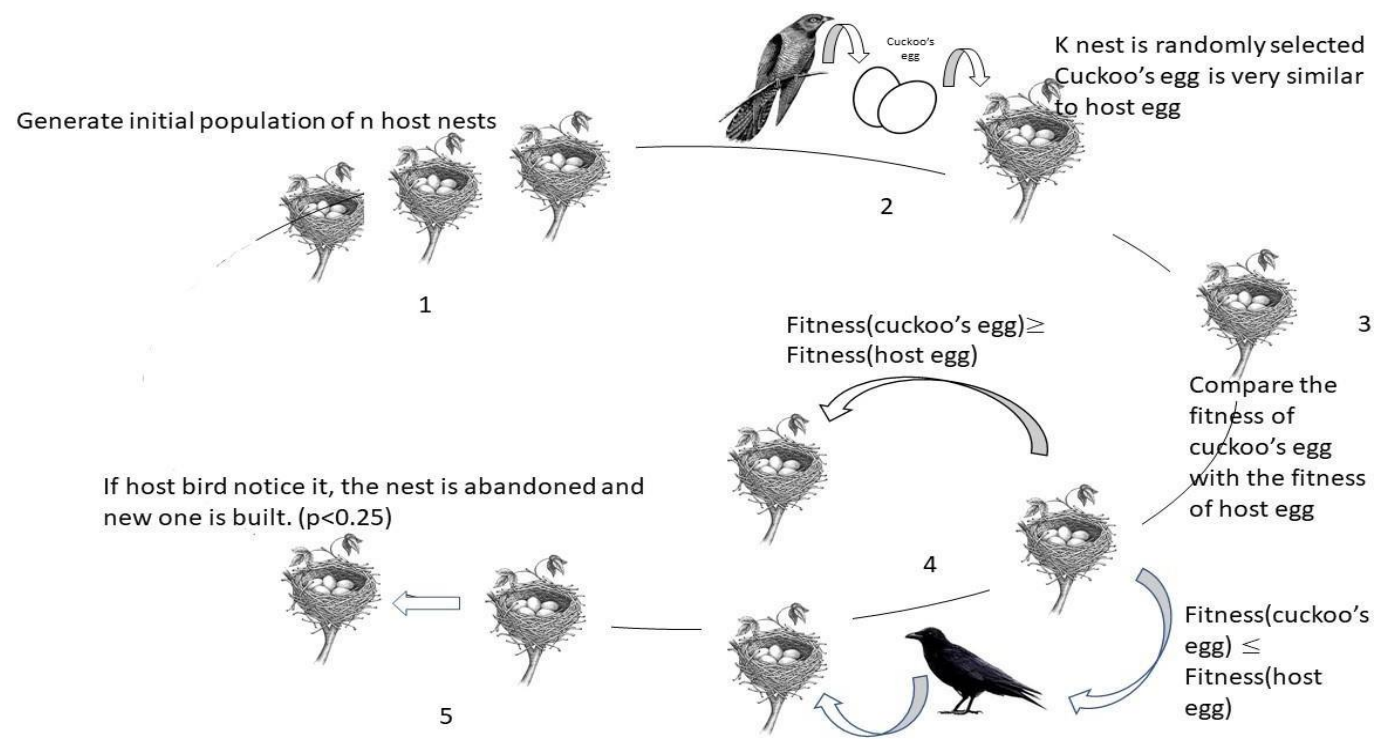

Figure 4. The behavior of cuckoo search.

\section{LEVY SEARCH ALGORITHM}

One of the important population-based algorithms is the levy flight algorithm. Utilize knowledge in the best possible way to solve any problem is the main job. LS algorithm is modified by subsuming PBA (population-based algorithm) in the LS algorithm. In this method, a global search helps to explore search space. Levy flight algorithm has been well utilized to find the most promising SP (search space). When the levy algorithm search in the search space at that time, it searches the surroundings also [21]. 
Brown et. al in the year 2007 first proposed the LSA algorithm inspiring from the natural tendency of insets and animals [22]. In the same year (2007), Pavlyukevich has used the algorithm [23]. Flying behavior has helped to improve the searching algorithm. In the search algorithm field, it gives a significant improvement [22-23].

The animal generally searches the prey randomly. The process of finding the prey is called random because depending on the transmission probability and current location, the next location is specified. The probability function is used to find the mathematical model for direction. LTP (levy tailed probability) and random walk by levy flight have a great impact on finding the distribution and step length.

Natural walking of levy flight is random, and steps are specified by step length. So, the distribution is found by levy has random step length. This mathematical model is mentioned below.

$$
(s) \sim|s|^{-1-\beta} \text {, where }(0<\beta \leq 2)
$$

's 'denotes the step length. For generating step size, the Mantegna algorithm has been used that helps the levy search a symmetric and stable distribution. Symmetric in the sense that it may be negative or positive. Step length s can calculate by using the Mantegna algorithm, which is described below.

$$
S=\frac{u}{|v|^{\frac{1}{\beta}}}
$$

' $u$ ' and ' $v$ ' can be derived from the normal distribution.

$$
u \sim N\left(0, \sigma_{u}^{2}\right), v \sim N\left(0, \sigma_{v}^{2}\right)
$$

Where

$$
\sigma_{u}=\left\{\frac{\Gamma(1+\beta) \sin \left(\frac{\pi \beta}{2}\right)}{\beta \Gamma\left[\frac{(1+\beta)}{2}\right] 2^{\frac{(\beta-1)}{2}}}\right\}^{\frac{1}{\beta}}, \quad \sigma_{v}=1
$$

's' complies with the levy distribution for $|s| \geq\left|s_{0}\right| . s_{0}$ denotes the SSL (smallest step length). $\Gamma$ (.) Represent the gamma function.

$$
\Gamma(1+\beta)=\int_{0}^{\infty} t^{\beta} e^{-t} d t .
$$

$\beta$ is an integer and $\Gamma(1+\beta)=\beta$. Step size is used to exploiting the search area. This is generated by levy distribution. Which is mentioned below.

$$
\text { step } \operatorname{size}(t)=0.001 \times s(t) \times S L C
$$

$\mathrm{T}$ is the iteration number at the time. By using equation (2) $\mathrm{s}(\mathrm{t})$ is calculated. In the global search algorithm, SLC represents the social learning component.

In LS strategy $i$ th individual is updated by equation (18).

$$
x_{i j}^{\prime}(t+1)=x_{i j}(t)+\operatorname{step}_{\text {size }(t)} \times U(0,1)
$$

\section{Levy flight Pseudocode}

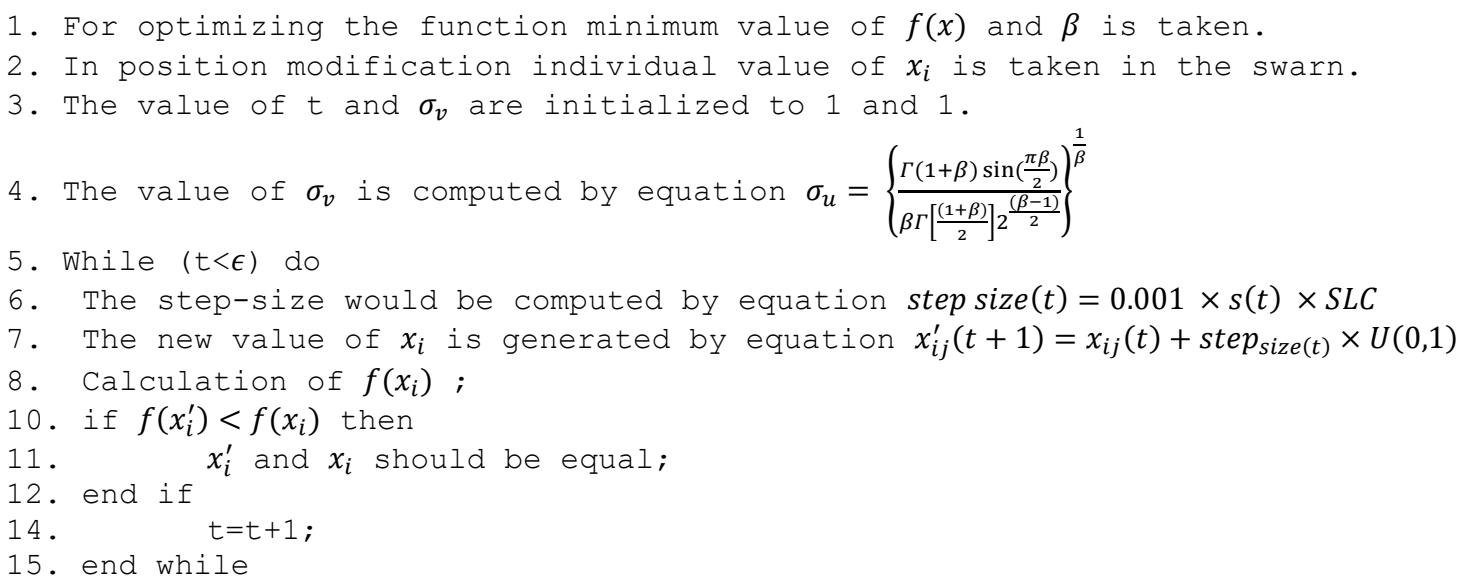

\section{PROPOSED MODIFIED GREY WOLF ALGORITHM}

Our proposed algorithm is based on one of the most popular metaheuristic optimization technique named the grey wolf optimization technique. The main problem of any straightforward optimization technique is local minimization and premature convergence. To overcome the premature convergence and local minimization, we modified the GWO algorithm and proposed the modified version in this article. This MGWO algorithm has been amalgamed with the Mantegna algorithm, CSA (cuckoo search algorithm), and levy fly search algorithm. Here the GWO algorithm is the same up to the initialization step. To find the unknown and known object cuckoo search algorithm has been introduced. Suitable fitness function (depending on the problem) helps to find the object (line 3.5 to 3.8 in the pseudocode) more precisely. 
To make the problem simple, inside the cuckoo search algorithm, we have tried to find one object in every iteration in this algorithm. Then the best three positioned where we get the best image have been taken. Depending on the average value of the three locations, the position of omega has been updated (lines 4 to 9 in the pseudocode). In our proposed algorithm levy flight search algorithm has been introduced to restrict the random movement (line 10.1 to 10.6 in the algorithm), and the step size of the algorithm has been modified using the Mantegna algorithm (line 10.6.1 to 10.6.2 in the pseudocode).

\section{Pseudocode: Modified Grey Wolf Optimization (MGWO)}

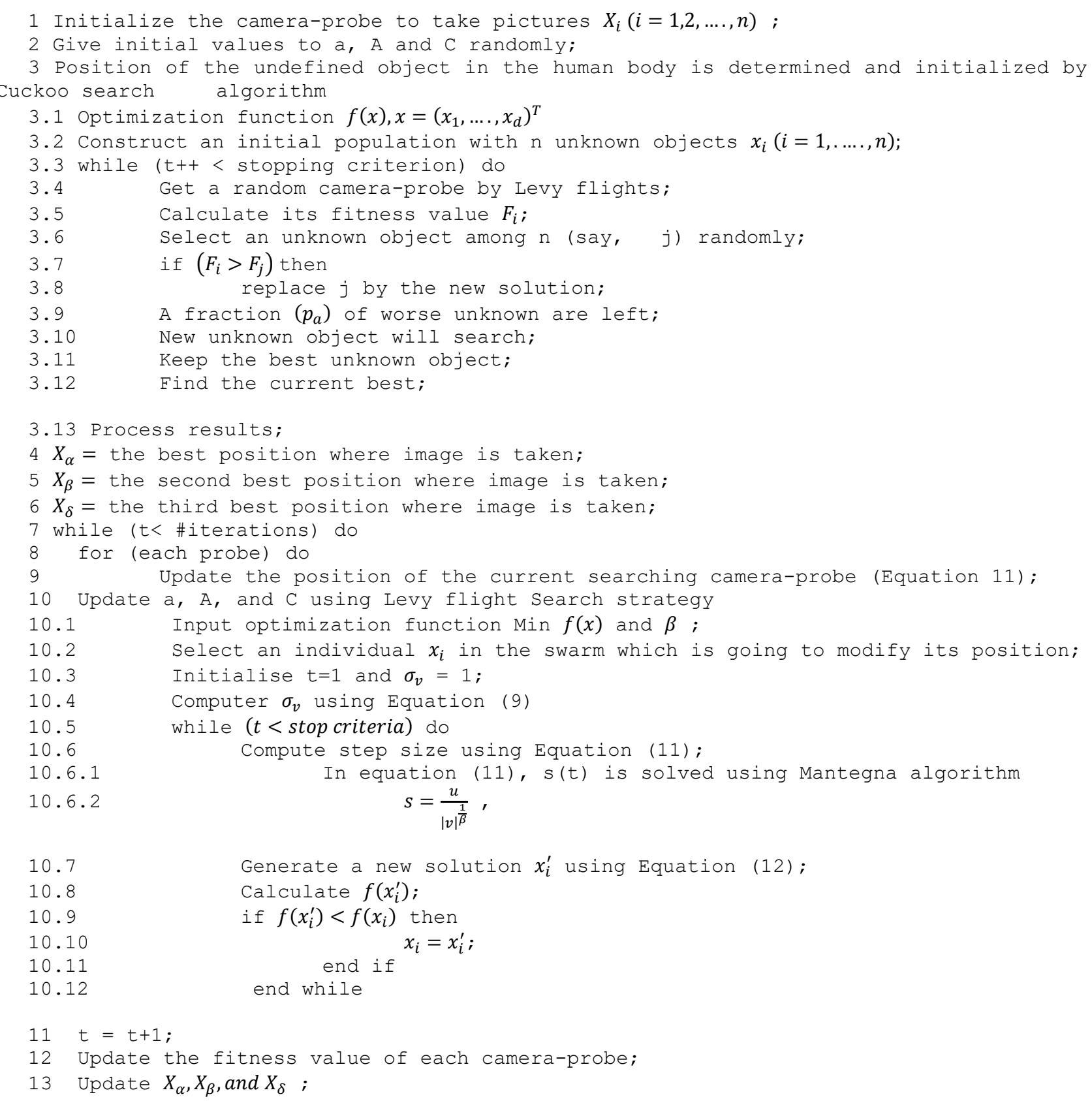


The flow diagram of our proposed MGWO is depicted in the figure below.

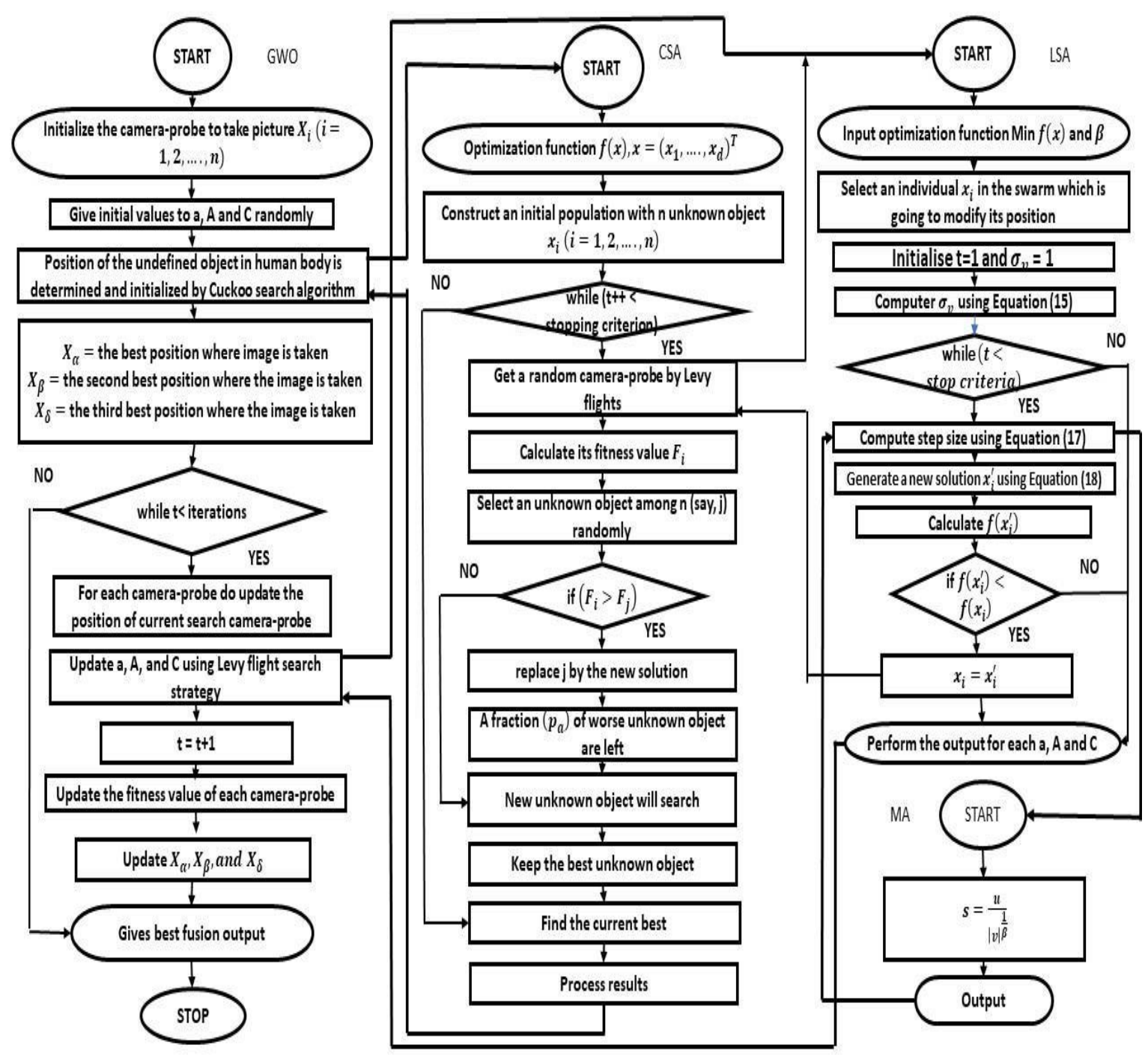

Figure 5. Flow diagram of Proposed Modified Grey Wolf Algorithm

\section{EXPERIMENTAL RESULTS}

Our own experimental result has been compared against CS-GWO, GWO, and CS algorithms. Here in this section, a two-step comparison has been depicted. At first, setting parameters has been mentioned then the algorithm is performed. In the next step, the implementation result has been mentioned. Table 3 and Table 4 gives the comparison percentage and comparison results. In this regard, the key features of those algorithms have been used.

Table 1. Setting of parameters

\begin{tabular}{|c|c|c|c|c|c|}
\hline $\begin{array}{c}\text { Description of } \\
\text { parameters }\end{array}$ & $\begin{array}{c}\text { MGWO } \\
\text { parameter } \\
\text { setting }\end{array}$ & $\begin{array}{c}\text { CS-GWO } \\
\text { parameter } \\
\text { setting }\end{array}$ & $\begin{array}{c}\text { GWO } \\
\text { parameter } \\
\text { setting }\end{array}$ & $\begin{array}{c}\text { CS parameter } \\
\text { setting }\end{array}$ & $\begin{array}{c}\text { Unselected } \\
\text { parameter } \\
\text { setting }\end{array}$ \\
\hline $\begin{array}{c}\text { Size of } \\
\text { population }\end{array}$ & 40 & 40 & 40 & 40 & 40 \\
\hline $\begin{array}{c}\text { Dimension of } \\
\text { problem }\end{array}$ & 40 & 40 & 40 & 40 & 40 \\
\hline $\begin{array}{c}\text { The maximum } \\
\text { iteration that } \\
\text { has been made }\end{array}$ & 500 & 40 & 40 & 500 & 40 \\
\hline $\begin{array}{c}\text { Total runs that } \\
\text { have been } \\
\text { made }\end{array}$ & 40 & & 500 & & \\
\hline
\end{tabular}


In the beginning, MGWO's parameters have been initialized. Setting parameters has been described in Table 1. In the first column of the table, the parameter description has been listed. In the parameter description row, GWO, MGWO, CS, CS-GWO, and the unselected algorithm has been described in the subsequent columns. In the next row size of the population is listed, followed by a description of parameters. The size of the population of GWO, MGWO, CS, CS-GWO, and the unselected algorithm is taken as 40 in each case. The dimension of the problem has been listed below the size of the population. The dimension of the problem in each case of GWO, MGWO, CS, CS-GWO, and the unselected algorithm is taken as 40. In the fourth row, the maximum iteration that has been taken is listed. The maximum is taken in each case of GWO, MGWO, CS, CS-GWO, and the unselected algorithm is 50. In the next column, the total run made by GWO, MGWO, CS, CS-GWO, and the unselected algorithm is listed. In each case the run has been made is 40 .

Table 2. Result after implementation of MGWO, CS-GWO, GWO, CS, and Unselected algorithm.

\begin{tabular}{|c|c|c|c|c|c|}
\hline & MGWO & CS-GWO & GWO & CS & Unselected \\
\hline $\begin{array}{c}\text { Number of } \\
\text { initial positions }\end{array}$ & 200 & 240 & 440 & 440 & 1600 \\
\hline $\begin{array}{c}\text { Number of } \\
\text { initial } \\
\text { populations }\end{array}$ & 40 & 40 & 40 & 40 & 40 \\
\hline $\begin{array}{c}\text { The ratio of } \\
\text { cross over }\end{array}$ & Dynamic & Dynamic & Dynamic & Dynamic & Static \\
\hline $\begin{array}{c}\text { Ration of } \\
\text { mutation }\end{array}$ & Dynamic & Dynamic & Dynamic & Dynamic & Static \\
\hline
\end{tabular}

The result of the MGWO algorithm is taken after applying the algorithm. The result of the implementation is listed in Table 2. The result of GWO, MGWO, CS, CS-GWO, and the unselected algorithm is listed in the consecutive columns. The number of initial positions is listed under row number 2 in Table 2. The number of initial positions of GWO and MGWO is 240 and 200. 440 and 240 are the number of initial position of CS and CS-GWO, respectively, and 1600 is the number of the initial positions for the unselected algorithm. In the next row number of the initial population is described. The initial population of GWO, MGWO, CS, CS-GWO, and unselected algorithms are 40 in each case. In the third row ratio of cross over is listed. The ration of crossover is dynamic for each case of GWO, MGWO, CS, and CS-GWO algorithm, where the ratio of the unselected algorithm is static. At the last row, the mutation ratio is described. The ratio of mutation is dynamic for each case of GWO, MGWO, CS, and CS-GWO algorithm, but the mutation ratio of the unselected algorithm is static.

Table 3. Comparison of percentage of optimization covered by MGWO against CS-GWO, GWO, CS, and Unselected algorithm.

\begin{tabular}{|c|c|c|}
\hline Algorithm & MGWO & CS-GWO \\
\hline $\begin{array}{l}\text { Total problem solved out of } \\
\qquad 15\end{array}$ & 9 out of 15 & 6 out of 15 \\
\hline At the age out of $100(\%)$ & $60 \%$ & $40 \%$ \\
\hline Algorithm & MGWO & GWO \\
\hline $\begin{array}{l}\text { Total problem solved out of } \\
15\end{array}$ & 10 out of 15 & 3 out of 15 \\
\hline At the age out of $100(\%)$ & $67 \%$ & $33 \%$ \\
\hline Algorithm & MGWO & $\mathrm{CS}$ \\
\hline Total problem solved out of & 9 out of 15 & 6 out of 15 \\
\hline At the age out of $100(\%)$ & $60 \%$ & $40 \%$ \\
\hline Algorithm & MGWO & Unselected \\
\hline $\begin{array}{l}\text { Total problem solved out of } \\
15\end{array}$ & 12 out of 15 & 3 out of 15 \\
\hline At the age out of $100(\%)$ & $80 \%$ & $20 \%$ \\
\hline
\end{tabular}

A detailed discussion about the superiority of MGWO is needed to discuss. So, in Table 3, a detailed comparison of MGWO with GWO, CS, CS-GWO, and unselected algorithm is described. In Table 3, the comparison of percentage optimization has been discussed. In the first row of the percentage optimization table, the comparison between MGWO and CS-GWO algorithm has been depicted. Here, in this case, MGWO can solve nine problems out of fifteen problems. On the other hand, CS-GWO can solve six problems out of fifteen problems. In terms of percentage covered, MGWO can cove $60 \%$ coverage where the CS-GWO can cover only $40 \%$. So, it is clear that MGWO is superior to the CS-GWO algorithm. In the second row, MGWO has been compared with GWO. Here MGWO can able to solve ten problems out of fifteen problems, but at the same time, the GWO algorithm is able to solve three problems out of fifteen problems. In terms of percentage, MGO and GWO algorithm can cover $67 \%$ and $33 \%$ coverage. In the next row comparison between 
MGWO algorithm and CS algorithm has been listed. Here nine problem out of fifteen problems has been solved by MGWO algorithm. At the same time, CS-GWO is able to solve six problems out of fifteen problems. The coverage of MGWO and CS-GWO is $60 \%$ and $40 \%$, respectively. In the fourth row, the comparison between the MGWO algorithm and CS algorithm has been described. In that case, the MGWO algorithm can able to solve twelve problems out of fifteen problems, but the unselected algorithm is able to solve only three problems out of fifteen problems. For easy understanding, if we describe it in terms of percentage, $80 \%$, and $20 \%$ are the coverage of MGWO and unselected algorithm. The comparative result of MGWO, CS-GWO, GWO, and CS is shown in Table 4.

Table 4. Comparative Results of MGWO

\begin{tabular}{|c|c|c|c|c|}
\hline Algorithm Name & $\begin{array}{l}\text { Original } \\
\text { Features } \\
\text { Number }\end{array}$ & $\begin{array}{l}\text { Subsets Features } \\
\text { Number }\end{array}$ & Accuracy & Best Fitness \\
\hline MGWO & 40 & 5 & $87.36 \%$ & $81.259 \%$ \\
\hline CS-GWO [24] & 40 & 6 & $83.54 \%$ & $78.811 \%$ \\
\hline GWO [24] & 40 & 11 & $83.54 \%$ & $75.561 \%$ \\
\hline CS [24] & 40 & 11 & $83.54 \%$ & $75.261 \%$ \\
\hline Unselected [24] & 40 & 40 & $77.15 \%$ & $55.107 \%$ \\
\hline
\end{tabular}

Proving the supremacy of the MGWO algorithm with GWO, CS, CS-GWO, and unselected algorithm, a comparison among GWO, MGWO, CS, CS-GWO, and the unselected algorithm has been made. So, in Table 4, a comparison among GWO, MGWO, CS, CS-GWO, and the unselected algorithm has been listed. The algorithm name is titled in the first column of the comparison result table. The original feature number is listed in the second column. In the third column feature number of the substrate is depicted. In the fourth and fifth columns, accuracy and best fitness are listed, respectively. It is clear from the above table that the fourth column of the unselected algorithm where no algorithm has been used is the worst performance in terms of accuracy and best fitness. CS algorithm is far better than the Unselected algorithm, which can be viewed from table 4. GWO algorithm is slightly superior to the CS algorithm if we compare in terms of fitness. CS-GWO is better than GWO, CS, and unselected algorithm, but MGWO gives the best result if we compare in terms of accuracy and fitness. MGWO is $4.57 \%$ better than CS-GWO, which is it's the nearest competitor, and MGWO is $3.1 \%$ superior to CS-GWO in terms of fitness. In this article, our proposed modified grey-wolf algorithm (which is incorporated with LSA, CS-GWO, MA, and CSA) is far superior to CS, CS-GWO, and GWO in terms of optimization.

\section{CONCLUSIONS}

In recent days, one of the most popular nature-inspired algorithms is GWO. The main cause of the popularity of GWO is due to local search strategy. It cannot provide better results in the global search case. This poor efficiency of GWO motivates us to explore in this domain. So, in this article, a novel metaheuristic algorithm of a modified grey wolf algorithm has been proposed. This proposed algorithm contains several efficient heuristic and metaheuristic algorithms to optimize image fusion. The proposed MGWO algorithm contains CSA and LSA for searching the effected cells and guiding the camera probe. This CS and LS algorithm optimizes the image fusion most accurate way. The trapping of local minima and premature convergence of GWO is overcome by judicious design strategy. The whole algorithm is optimized using GWO, CSA, LSA, MA algorithm, thus lowering the approximation. We have extensively compared the performance of MGWO with respect to CS-GWO, GWO, CA, and unselected algorithms. The quality of the proposed synthesized architecture is successfully assessed by simulation using ModelSim and also on-board testing in the case of real-time application.

\section{REFERENCES}

[1] Mirjalili, S., Mirjalili, S. M., \& Lewis, A. (2014). Grey wolf optimizer. Advances in engineering software, 69, 46- 61.

[2] Daniel, E., Anitha, J., Kamaleshwaran, K. K., \& Rani, I. (2017). Optimum spectrum mask based medical image fusion using Gray Wolf Optimization. Biomedical Signal Processing and Control, 34, 36-43.

[3] Daniel, E., Anitha, J., \& Gnanaraj, J. (2017). Optimum laplacian wavelet mask based medical image using hybrid cuckoo search-grey wolf optimization algorithm. Knowledge-Based Systems, 131, 58-69.

[4] Daniel, E. (2018). Optimum wavelet-based homomorphic medical image fusion using hybrid genetic-grey wolf optimization algorithm. IEEE Sensors Journal, 18(16), 6804-6811.

[5] Alfailakawi, M. G., El-Shafei, M., Ahmad, I., \& Salman, A. (2018). FPGA-based implementation of cuckoo search. IET Computers \& Digital Techniques, 13(1), 28-37.

[6] Jia, R. M., \& He, D. X. (2013, July). Complex valued cuckoo search with local search. In 2013 Ninth International Conference on Natural Computation (ICNC) (pp. 1804-1808). IEEE. 
[7] Fu, G. P., Hong, S. H., Li, F. L., \& Wang, L. (2020). A novel multi-focus image fusion method based on distributed compressed sensing. Journal of Visual Communication and Image Representation, 67, 102760.

[8] Zhang, K., Huang, Y., Yuan, X., Ma, H., \& Zhao, C. (2020). Infrared and visible image fusion based on intuitionistic fuzzy sets. Infrared Physics \& Technology, 105, 103124.

[9] Zheng, M., Qi, G., Zhu, Z., Li, Y., Wei, H., \& Liu, Y. (2020). Image Dehazing by An Artificial Image Fusion Method based on Adaptive Structure Decomposition. IEEE Sensors Journal.

[10] Li, C., Tang, S., Yan, J., \& Zhou, T. (2020). Low-light image enhancement via pair of complementary gamma functions by fusion. IEEE Access, 8, 169887-169896.

[11] Wong, L. I., Sulaiman, M. H., Mohamed, M. R., \& Hong, M. S. (2014). Grey Wolf Optimizer for solving economic dispatch problems. In 2014 IEEE International Conference on Power and Energy (PECon) (pp. 150-154). IEEE.

[12] Gupta, P., Rana, K. P. S., Kumar, V., Mishra, P., Kumar, J., \& Nair, S. S. (2015). Development of a Grey Wolf Optimizer Toolkit in LabVIEWTM. In 2015 International Conference on Futuristic Trends on Computational Analysis and Knowledge Management (ABLAZE) (pp. 107-113). IEEE.

[13] Vosooghifard, M., \& Ebrahimpour, H. (2015). Applying Grey Wolf Optimizer-based decision tree classifer for cancer classification on gene expression data. In 2015 5th International Conference on Computer and Knowledge Engineering (ICCKE) (pp. 147-151). IEEE.

[14] Yang, X. S., \& Deb, S. (2014). Cuckoo search: recent advances and applications. Neural Computing and Applications, 24(1), 169-174.

[15] Alfailakawi, M. G., El-Shafei, M., Ahmad, I., \& Salman, A. (2018). FPGA-based implementation of cuckoo search. IET Computers \& Digital Techniques, 13(1), 28-37.

[16] Girsang, A. S., Yunanto, A., \& Aslamiah, A. H. (2017). A hybrid cuckoo search and K-means for clustering problem. In 2017 International Conference on Electrical Engineering and Computer Science (ICECOS) (pp. 120-124). IEEE.

[17] Jia, R. M., \& He, D. X. (2013). Complex valued cuckoo search with local search. In 2013 Ninth International Conference on Natural Computation (ICNC) (pp. 1804-1808). IEEE.

[18] Nguyen, K. P., \& Fujita, G. (2017). Multi-area economic dispatch in bulk system using self-learning cuckoo search algorithm. In 2017 52nd International Universities Power Engineering Conference (UPEC) (pp. 1-6). IEEE.

[19] Zefan, C., \& Xiaodong, Y. (2017). Cuckoo search algorithm with deep search. In 2017 3rd IEEE International Conference on Computer and Communications (ICCC) (pp. 2241-2246). IEEE.

[20] Zhang, M., Zhu, Z., \& Cui, Z. (2017). Weighted-based oriented cuckoo search. In 2017 9th International Conference on Modelling, Identification and Control (ICMIC) (pp. 365-369). IEEE.

[21] Sharma, H., Bansal, J. C., Arya, K. V., \& Yang, X. S. (2016). Lévy flight artificial bee colony algorithm. International Journal of Systems Science, 47(11), 2652-2670.

[22] Brown, C. T., Liebovitch, L. S., \& Glendon, R. (2007). Lévy flights in Dobe Ju/'hoansi foraging patterns. Human Ecology, 35(1), 129-138.

[23] Pavlyukevich, I. (2007). Lévy flights, non-local search and simulated annealing. Journal of Computational Physics, 226(2), 1830-1844.

[24] Xu, H., Liu, X., \& Su, J. (2017). An improved grey wolf optimizer algorithm integrated with Cuckoo Search. In 20179 th IEEE international conference on intelligent data acquisition and advanced computing systems: technology and applications (IDAACS) (Vol. 1, pp. 490-493). IEEE 